ISSN 0103-9954

\title{
INFLUÊNCIA DA DISPOSIÇÃO DOS TUBETES E DA APLICAÇÃO DE FERTILIZANTES DE LIBERAÇÃO LENTA, DURANTE O PRÉ-VIVEIRO, NO CRESCIMENTO DE MUDAS DE DENDEZEIRO (Elaeis guineensis Jacq.) ${ }^{1}$
}

\section{CONTAINER DISTRIBUTION AND SLOW RELEASE FERTILIZERS APPLICATION ALONG THE PRE-NURSERY INFLUENCING OIL PALM SEEDLINGS GROWTH}

\author{
Paulo César Teixeira ${ }^{2}$ Haroldo Silva Rodrigues ${ }^{3}$ Wanderlei Antônio Alves Lima ${ }^{4}$ \\ Raimundo Nonato Carvalho Rocha ${ }^{5}$ Raimundo Nonato Vieira Cunha ${ }^{4}$ Ricardo Lopes ${ }^{4}$
}

\section{RESUMO}

Este trabalho objetivou verificar a influência do tipo e doses de adubo de liberação lenta (ALL) e da percentagem de ocupação da bandeja pelos tubetes $(\mathrm{OB})$, durante o pré-viveiro, no crescimento, nutrição e partição de matéria seca de mudas de dendezeiro. O experimento foi constituído de 16 tratamentos, em esquema fatorial, compreendendo dois tipos de ALL (Osmocote ${ }^{\circledR}$ e Basacote mini), duas doses $\left(0\right.$ e $3 \mathrm{~kg} / \mathrm{m}^{3}$ de substrato) e quatro $\mathrm{OB}(100 \%, 66 \%, 50 \%$ e $25 \%)$, no delineamento em blocos casualizados, com quatro repetições. Ainda, houve um tratamento adicional em sacos plásticos contendo $1,5 \mathrm{dm}^{3}$ de solo. Sementes pré-germinadas de dendezeiro foram colocadas em tubetes contendo substrato comercial e em sacos contendo solo. Aos três meses, todas as mudas foram transplantadas para sacos plásticos de $40 \times 40 \mathrm{~cm}$ contendo solo avaliando a altura de planta e diâmetro do coleto, a matéria seca e o teor de N, P, K, Ca e de Mg. Aos 10 e 16 meses de idade, foram avaliados a altura e diâmetro e aos 16 meses a matéria seca. A adição de ALL no pré-viveiro foi fundamental para a formação final das mudas. A OB no pré-viveiro não influenciou o crescimento em altura e diâmetro aos 10 e 16 meses de idade. A altura das mudas produzidas em tubetes foi superior, aos 10 meses de idade, com o Osmocote ${ }^{\circledR}$, comparada ao Basacote mini. Aos 16 meses, não houve diferença de crescimento entre as plantas com os diferentes tipos de ALL, havendo somente efeito da dose utilizada.

Palavras-chave: dendê; produção de mudas; adubação; espaçamento.

\section{ABSTRACT}

This research had as objective to verify the influence in growth, nutrition and dry matter partition in oil palm seedling by type and dosages of slow release fertilizers (SRF) and percentage of tray occupation by plastic containers during pre-nursery. The experiment consisted of 16 treatments, in factorial scheme: two types of SRF (Osmocote ${ }^{\circledR}$ e Basacote mini), two dosages $\left(0\right.$ and $\left.3 \mathrm{~kg} / \mathrm{m}^{3}\right)$ and four schemes for the container distribution used to attain $100 \%, 66 \%, 50 \%$ and $25 \%$ of tray occupation. An additional treatment composed of $15 \times 15 \mathrm{~cm}$ plastic bags filled with soil was added. Pre-germinated seeds of oil palm were put in plastic containers of $120 \mathrm{~cm}^{3}$ containing substratum and in plastic bags containing soil. After three months, the seedlings were transplanted to $40 \times 40 \mathrm{~cm}$ plastic bags containing soil. At this time, height, diameter, dry matter and concentration of $\mathrm{N}, \mathrm{P}, \mathrm{K}, \mathrm{Ca}$ and $\mathrm{Mg}$ were evaluated. After 10 months, seedlings were evaluated for height and diameter and after 16 months, seedlings had the height, diameter and dry matter weight evaluated. Addition of SRF was fundamental for seedlings development. Different percentages of tray occupation by containers during pre-nursery did not influence height and diameter of oil palm seedlings at 10 and 16 months old. The evaluation after 10 months showed that plants fertilized with Osmocote ${ }^{\circledR}$ were higher than those fertilized with Basacote mini. The evaluations after 16 months showed that plants fertilized during the pre-nursery had higher height, diameter and leaflets, leaf, aboveground and total dry matter than

1. Apoio financeiro do CNPq e Embrapa Transferência de Tecnologia/Escritório de Negócios da Amazônia.

2. Engenheiro Agrônomo, Dr., Pesquisador da Embrapa Amazônia Ocidental, Rod. AM 010, km 29, Caixa Postal 319 , CEP 69010-960, Manaus (AM). paulo.teixeira@cpaa.embrapa.br

3. Estudante de Agronomia, Universidade Federal do Amazonas, bolsista CNPq de Iniciação Científica na Embrapa Amazônia Ocidental, Rod. AM 010, km 29, Caixa Postal 319, CEP 69010-960, Manaus (AM).

4. Engenheiros Agrônomos, Dr., Pesquisadores da Embrapa Amazônia Ocidental, Rod. AM 010, km 29, Caixa Postal 319, CEP 69010-960, Manaus (AM).

5. Engenheiro Agrônomo, Dr., Analista da Embrapa Amazônia Ocidental, Rod. AM 010, km 29, Caixa Postal 319 , CEP 69010-960, Manaus (AM).

Recebido para publicação em 12/11/2007 e aceito em 11/05/2009. 
plants not fertilized.

Keywords: seedling production; fertilization; spacing.

\section{INTRODUÇÃO}

O dendezeiro (Elaeis guineensis Jacq.) é uma planta perene tropical de origem africana que expressa melhor seu potencial de produção em condições de alta temperatura, boa radiação solar, alta precipitação e umidade relativa do ar, por isso, as principais áreas de cultivo estão localizadas nas regiões tropicais úmidas na África, Ásia e América.

A fase de produção de mudas tem como objetivo a obtenção de plantas de alta qualidade e em condições para serem levadas ao campo na época apropriada. Em um empreendimento, o sucesso de um plantio de dendezeiro muito se deve ao processo adotado na formação de mudas e a sua qualidade se reflete diretamente na precocidade e na maior produção na fase jovem, assim como no maior potencial de produção na fase adulta (PACHECO e TAILLIEZ, 1985; BARCELOS et al., 2001).

A formação de mudas de dendezeiro normalmente é feita em duas fases denominadas normalmente de pré-viveiro e de viveiro. O pré-viveiro é a denominação dada à fase de formação da muda em área coberta com sombrite ou palha de palmeiras e o viveiro, normalmente em área a céu aberto. A forma tradicional de produção de mudas de dendezeiro durante o pré-viveiro (até aproximadamente 3-4 meses de idade) é a utilização de sacos plásticos contendo amostras de solo (PACHECO e TAILLIEZ, 1985; BARCELOS et al., 2001; CORLEY e TINKER, 2003), mas esse processo demanda a necessidade de grandes áreas de préviveiro, grande necessidade de mão-de-obra para o manejo das mudas e existe a possibilidade de rompimento dos recipientes. A tendência para várias culturas é a substituição dos sacos plásticos pelos tubetes de plástico rígido (exemplos, JINKS e MASON, 1998; SCHWENGBER et al., 2002; YAMANISHI et al., 2004; DOMINGUEZ-LERENA et al., 2006; FREITAS et al., 2006) e, recentemente, esta técnica tem sido estudada para a formação de mudas de dendezeiro (CHEE et al., 1997; TEIXEIRA et al., 2006). Algumas vantagens técnicas do sistema de tubetes para a formação de mudas florestais são citadas por Simões (1987) e Gonçalves et al. (2000), entre as quais, destacam-se: boa formação do sistema radicular, rápido crescimento inicial das mudas, menor consumo de substrato, maior número de mudas por unidade de área, possibilidade de reciclagem e facilidades operacionais, como o transporte das mudas e o peso quando comparado ao sistema convencional de produção de mudas em sacos plásticos. Segundo Chee et al. (1997), o crescimento no campo de mudas de dendezeiro produzidas em tubetes durante o pré-viveiro pode ser tão bom quanto o de mudas crescidas em sacos plásticos. A utilização dessa metodologia, entretanto, ainda carece de estudos, pois são poucos os trabalhos relacionados, sobretudo para a cultura do dendezeiro.

O adubo é um fator importante na formação das mudas em tubetes, pois deverá corrigir a perda de nutrientes do substrato por lixiviação da água e disponibilizar também os nutrientes necessários para as mudas. As pequenas dimensões dos tubetes e, consequentemente, o pequeno volume de substrato que suportam, exigem, entretanto, aplicação de doses altas de nutrientes solúveis, em razão das perdas por lixiviação, resultantes da necessidade de regas freqüentes (NEVES et al., 1990). Entretanto, doses altas de fertilizantes solúveis, sobretudo na fertilização de base, elevam a concentração salina do substrato, podendo causar falhas de germinação, distúrbios nutricionais e, conseqüentemente, retardamento do crescimento inicial das mudas (GONÇALVES et al., 2000). Adubos de liberação lenta e controlada (ALL) promovem liberação contínua de nutrientes em função do tempo, o que elimina a necessidade de freqüentes adubações, sincronizando a demanda da planta com a disponibilidade de nutrientes no substrato.

Atualmente, os ALL vêm sendo testados na produção de mudas de várias espécies em recipientes (OLIVEIRA et al. 1995; ANDRADE NETO et al., 1999; SIDHU e SINURAYA, 2000). Oliveira et al. (1995) testaram o efeito de diferentes doses de ALL (fórmula 17-9-13, adicionado ao substrato comercial) na produção de mudas de cafeeiro e verificaram que aquelas que receberam o formulado de liberação lenta de nutrientes apresentaram maior altura, alto vigor, melhor sanidade e bom aspecto visual, além de diminuição de 40 dias na formação das mesmas e considerável economia de mão-de-obra. No entanto, são raros os trabalhos que comprovam a eficiência deste adubo na produção de mudas de dendezeiro, especialmente em fase de pré-viveiro, necessitando, portanto, de mais pesquisas.

Além da fertilização, o espaçamento entre as mudas no viveiro é um dos fatores importantes que influenciam o crescimento (CHINCHILLA et al., 1990) e isso ainda carece de estudos relacionados à 
produção de mudas de dendezeiro em tubetes na fase de pré-viveiro. De maneira geral, as espécies constituintes de qualquer sistema de plantio apresentam diferentes exigências por luz, água e nutrientes, e o estudo do crescimento das mudas nos tubetes, em diferentes densidades de plantio, torna-se necessário para o entendimento de seu comportamento, especialmente com relação à competição por luz, contribuindo desta forma para o conhecimento dos mecanismos relacionados ao uso dos recursos primários. A interceptação da radiação fotossinteticamente ativa pelas culturas exerce grande influência quando outros fatores ambientais são favoráveis (OTTMAN e WELCH, 1989).

Este trabalho teve como objetivos verificar a influência da utilização de adubos de liberação lenta e da taxa de ocupação da bandeja pelos tubetes, durante a fase de pré-viveiro, na formação de mudas de dendezeiro.

\section{MATERIAL E MÉTODOS}

Este trabalho foi conduzido na Embrapa Amazônia Ocidental, inicialmente em casa de sombra (sombrite 50\%) localizada no $\mathrm{km} 29$, Rodovia AM 010, em Manaus, AM, durante a fase de pré-viveiro, e em céu aberto (viveiro), no campo experimental do Rio Urubu, no município de Rio Preto da Eva, AM.

Sementes de dendezeiro, do híbrido intraespecífico comercial Tenera, foram colocadas para germinar pelo método do calor seco (NUNES et al., 1998). As sementes pré-germinadas foram colocadas em tubetes plásticos de $120 \mathrm{~cm}^{3}$ contendo o substrato comercial e condicionador de solo farelado "Germina Plant" (para horta, com $10 \%$ de turfa e $102 \%$ de capacidade de retenção de água) com a seguinte composição química: $\mathrm{pH}$ $\left(\mathrm{H}_{2} \mathrm{O}\right): 5,2$; M.O.: $441 \mathrm{~g} / \mathrm{dm}^{3}, \mathrm{P}: 49 \mathrm{mg} / \mathrm{dm}^{3}, \mathrm{~K}: 3,3 \mathrm{mmol}_{\mathrm{c}} / \mathrm{dm}^{3}, \mathrm{Ca}: 350 \mathrm{mmol}_{\mathrm{c}} / \mathrm{dm}^{3}, \mathrm{Mg}: 100 \mathrm{mmol} / \mathrm{dm}^{3}$, $\mathrm{H}+\mathrm{Al}: 47 \mathrm{mmol}_{\mathrm{c}} / \mathrm{dm}^{3}, \mathrm{SB}: 453 \mathrm{mmol}_{\mathrm{c}} / \mathrm{dm}^{3}$, T: $500 \mathrm{mmol}_{\mathrm{d}} / \mathrm{dm}^{3}, \mathrm{~V}: 91 \%$.

O experimento foi constituído de 16 tratamentos, em blocos casualizados, com quatro repetições, em esquema fatorial $2^{2} \times 4$, compreendendo dois tipos de ALL: a) Osmocote ${ }^{\circledR}$ - formulação 15-09-12 e Mg: 1\%, S: 2,3\%, B: 0,02\%, Cu: 0,05\%, Fe: $1 \%$, Mn: 0,06\%, Mo: 0,02\%, Zn: 0,05\%, com período de liberação total dos nutrientes de aproximadamente 5-6 meses, e b) Basacote mini - formulação 13-06-16 e Mg: 1,4\%, S: $10 \%$, B: $0,02 \%, \mathrm{Cu}: 0,05 \%$ u, Fé: $0,26 \%$ e, Mn: 0,06\%n, Mo:0,02\%, com período de liberação total dos nutrientes de aproximadamente três meses; duas doses dos ALL ( 0 e $3 \mathrm{~kg} / \mathrm{m}^{3}$ de substrato); e quatro taxas de ocupação da bandeja pelos tubetes $(100,66,50$ e 25\%) (Figura 1). Além destes, houve um tratamento adicional composto de sacos plásticos de $15 \times 15 \mathrm{~cm}$ contendo aproximadamente $1,5 \mathrm{dm}^{3}$ de amostras de Latossolo Amarelo argiloso (terriço) sem adubação (método tradicional). Cada unidade experimental (repetição) no pré-viveiro foi constituída de 16 mudas.

Todas as mudas permaneceram na fase de pré-viveiro sob sombrite $(50 \%)$ por três meses. Nesse período, nenhuma adubação adicional foi feita. Sempre que necessário, foi feita a irrigação e a limpeza manual das plantas invasoras.

Aos três meses de idade, todas as mudas foram levadas para o Campo Experimental do Rio Urubu (CERU), no município de Rio Preto da Eva, AM, e transplantadas para sacos plásticos de $40 \mathrm{x} 40 \mathrm{~cm}$ contendo amostras de Latossolo Amarelo argiloso originárias do CERU. As amostras de solo foram coletadas na camada superficial $(0-10 \mathrm{~cm})$, destorroadas e passadas em peneira de malha de $4 \mathrm{~mm}$. Os sacos utilizados possuíam furos para permitir o escoamento do excesso de água.

Para o transplante, foi feito uma cova no centro dos sacos, utilizando o próprio tubete como broca. Uma muda foi colocada na cova e o espaço restante foi preenchido de modo que o coleto das plantas permanecesse no mesmo nível do solo, após ligeira compactação. Aproximadamente $0,5 \mathrm{~kg} / \mathrm{sacola}$ de casca (endocarpo) de sementes de dendezeiro foram colocados superficialmente como cobertura morta em todos os tratamentos. As mudas transplantadas foram dispostas no viveiro em triângulo eqüilátero, sendo a distância entre os sacos de $1 \mathrm{~m}$ na linha e $0,85 \mathrm{~m}$ na entrelinha. Cada unidade experimental no viveiro foi constituída de dez sacos, ou seja, de dez mudas. 


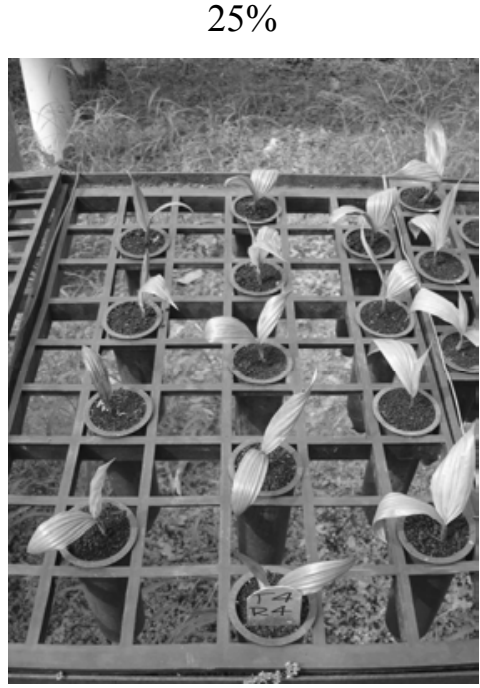

$66 \%$

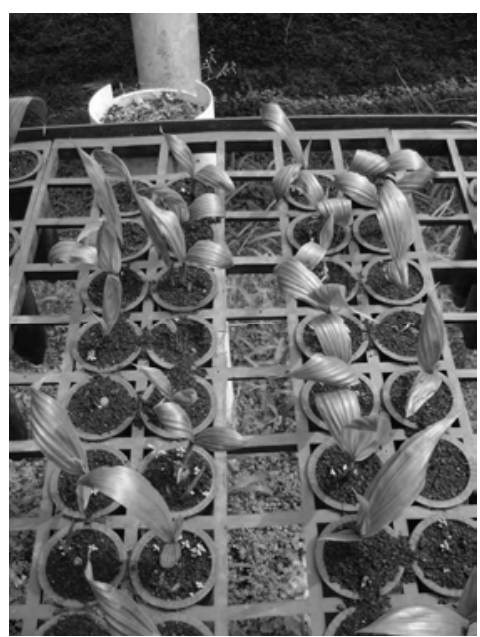

$50 \%$

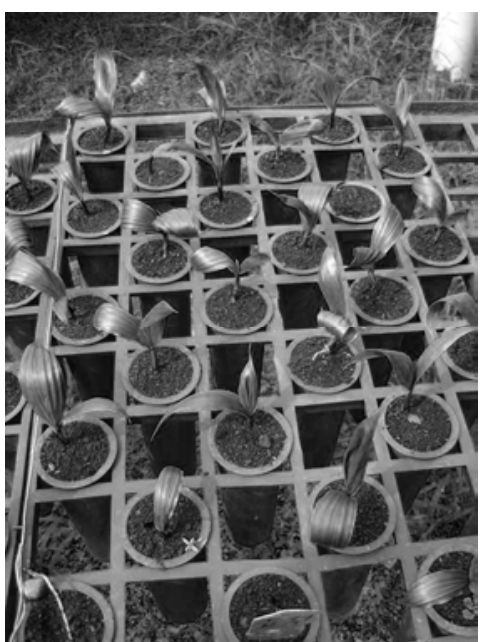

$100 \%$

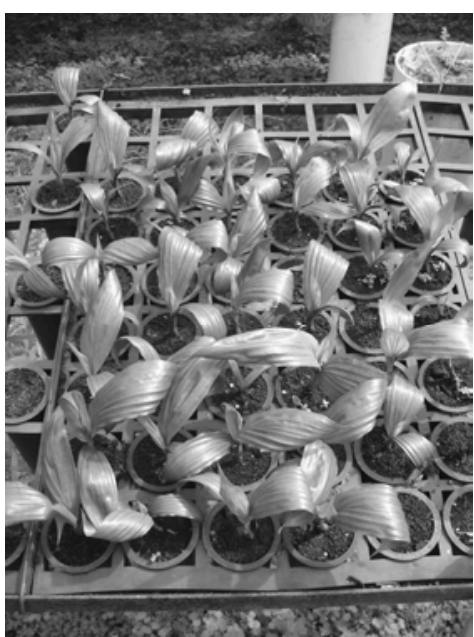

FIGURA 1: Esquema da distribuição dos tubetes nas bandejas.

FIGURE 1: Schemes of the distribution of the containers in the tray.

No momento do transplante das mudas, foi feita a medição da altura e do diâmetro do coleto de seis mudas e, a seguir, estas mudas foram cortadas. O material vegetal colhido foi separado em parte aérea e raízes. As raízes foram retiradas do substrato e do solo mediante lavagem com água corrente, sobre peneira de $0,5 \mathrm{~mm}$ de malha e, a seguir, passadas em água destilada.

O material vegetal colhido foi acondicionado em sacos de papel e colocado em estufa de circulação forçada de ar a $65^{\circ} \mathrm{C}$ por $72 \mathrm{~h}$. O processamento das amostras e a determinação dos teores de N, P, K, Ca e de Mg seguiram metodologias descritas por Malavolta et al. (1997).

No viveiro, todas as plantas receberam adubação mensal a partir do momento do transplante (três meses) com quantidades de adubos conforme sugeridas por Barcelos et al. (2001). Foi feita também uma adubação foliar mensal com solução de sulfato de cobre, na base de $30 \mathrm{~g} / 100 \mathrm{~L}$ de água no quarto mês e de $50 \mathrm{~g} / 100 \mathrm{~L}$ do quinto ao décimo-sexto mês.

Aos 10 e 16 meses de idade, foi avaliada a altura e o diâmetro do coleto das plantas com o auxílio de uma trena e um paquímetro digital, respectivamente. Aos 16 meses, as mudas foram cortadas e separadas em folíolos, pecíolo mais ráquis, caule e raízes. As raízes foram separadas do solo mediante lavagem em água corrente sob peneira de malha de $2 \mathrm{~mm}$. O material vegetal colhido foi acondicionado em sacos de papel e colocado em estufa de circulação forçada de ar a $65^{\circ} \mathrm{C}$ por $72 \mathrm{~h}$ e, a seguir, pesado. 
Os dados obtidos foram submetidos à análise de variância e testes de médias (Tukey, $\mathrm{p}<0,05$ ).

\section{RESULTADOS E DISCUSSÃO}

Os dados obtidos para crescimento em altura, diâmetro do coleto e produção de matéria seca durante a fase de pré-viveiro (até os três meses de idade) encontram-se na Tabela 1. Verificou-se que a dose do adubo de liberação lenta (ALL) foi o fator que mais influenciou esta fase e foi significativo para todas as variáveis analisadas (Tabela 2). O fator ocupação da bandeja somente foi significativo para matéria seca de raízes (MSR) e total (MST). Jinks e Mason (1998) verificaram que o aumento na densidade na produção de mudas reduziu a biomassa de raízes e da parte aérea. Timmis e Tanaka (1976) afirmam que a diminuição da produção de biomassa em densidades maiores é causada por redução considerável na interceptação da radiação fotossinteticamente ativa que pode ser até dez vezes menor em maiores densidades. Neste trabalho, quando foram utilizados os ALL, das cinco variáveis analisadas, apenas para matéria seca da parte aérea (MSPA) com o Basacote mini foram verificadas diferenças estatisticamente significativas entre as densidades de ocupação da bandeja, indicando, portanto, que as maiores densidades não diminuíram significativamente a produção de biomassa. O tipo do ALL somente influenciou o crescimento em diâmetro e a MST (Tabela 1).

De modo geral, as mudas produzidas em tubetes e adubadas com os ALL apresentaram crescimento e produção de matéria seca médios superiores às produzidas em tubetes sem adubação, independente da percentagem de ocupação das bandejas (Tabela 1).

TABELA 1: Diâmetro do coleto, altura, matéria seca da parte aérea (MSPA), das raízes (MSR) e total (MST) de mudas de dendezeiro (Elaeis guineensis Jacq.), aos três meses de pré-viveiro, em função do tipo (TA) e doses de adubo de liberação lenta e da percentagem de ocupação das bandejas pelos tubetes $(\mathrm{OB})$.

TABLE 1: Diameter, height, aboveground, roots and total dry matter of oil palm seedlings (Elaeis guineensis Jacq.), evaluated after three months of pre-nursery, in response to the type (TA) and dosages of slow release fertilizer and of percentage of tray occupation by containers.

\begin{tabular}{|c|c|c|c|c|c|c|c|}
\hline \multirow{2}{*}{ TA } & \multirow{2}{*}{$\begin{array}{l}\text { Dose } \\
\mathrm{kg} / \mathrm{m}^{3}\end{array}$} & \multirow{2}{*}{$\begin{array}{l}\text { OB } \\
\%\end{array}$} & \multirow{2}{*}{$\begin{array}{c}\text { Diâmetro } \\
\mathrm{mm}\end{array}$} & \multirow{2}{*}{$\begin{array}{c}\text { Altura } \\
\mathrm{cm}\end{array}$} & MSPA & MSR & MST \\
\hline & & & & & \multicolumn{3}{|c|}{ g/planta } \\
\hline - & 0 & 100 & $6,86 \mathrm{a}$ & $18,48 \mathrm{ab}$ & $4,24 \mathrm{a}$ & $3,20 \mathrm{a}$ & $7,44 a b$ \\
\hline- & 0 & 66 & $6,43 a$ & $16,35 \mathrm{~b}$ & $3,42 \mathrm{a}$ & $1,76 a$ & $5,18 \mathrm{~b}$ \\
\hline - & 0 & 50 & $6,90 \mathrm{a}$ & $21,70 \mathrm{a}$ & $4,89 \mathrm{a}$ & $3,45 \mathrm{a}$ & $8,34 a$ \\
\hline \multirow[t]{2}{*}{ - } & 0 & 25 & $5,92 \mathrm{a}$ & $17,73 \mathrm{ab}$ & $3,56 \mathrm{a}$ & $2,74 \mathrm{a}$ & $6,30 \mathrm{ab}$ \\
\hline & & Média & $6,53 \mathrm{C}$ & $18,56 \mathrm{~B}$ & $4,03 \mathrm{C}$ & $2,79 \mathrm{C}$ & $6,82 \mathrm{C}$ \\
\hline \multirow{5}{*}{ Osmocote ${ }^{\circledR}{ }^{1 /}$} & 3 & 100 & $7,55 \mathrm{a}$ & $24,60 \mathrm{a}$ & $7,39 a$ & $2,74 a$ & $10,13 a$ \\
\hline & 3 & 66 & $7,45 \mathrm{a}$ & $24,85 \mathrm{a}$ & $7,98 \mathrm{a}$ & $3,27 \mathrm{a}$ & $11,25 \mathrm{a}$ \\
\hline & 3 & 50 & $8,95 \mathrm{a}$ & $24,05 \mathrm{a}$ & $8,13 a$ & $3,48 \mathrm{a}$ & $11,60 \mathrm{a}$ \\
\hline & 3 & 25 & $8,00 \mathrm{a}$ & $23,30 \mathrm{a}$ & $6,52 \mathrm{a}$ & $4,51 \mathrm{a}$ & $11,03 \mathrm{a}$ \\
\hline & & Média & $7,99 \mathrm{~B}$ & $24,20 \mathrm{~A}$ & $7,50 \mathrm{~B}$ & $3,50 \mathrm{~B}$ & $11,00 \mathrm{~B}$ \\
\hline \multirow{5}{*}{ Basacote mini $^{2 /}$} & 3 & 100 & $9,20 \mathrm{a}$ & $25,22 \mathrm{a}$ & $9,49 a$ & $4,19 \mathrm{a}$ & $13,68 \mathrm{a}$ \\
\hline & 3 & 66 & $9,26 \mathrm{a}$ & $25,85 \mathrm{a}$ & $9,07 \mathrm{a}$ & $4,12 \mathrm{a}$ & $13,18 \mathrm{a}$ \\
\hline & 3 & 50 & $8,36 \mathrm{a}$ & $23,48 \mathrm{a}$ & $6,78 \mathrm{~b}$ & $5,19 a$ & $11,97 \mathrm{a}$ \\
\hline & 3 & 25 & $8,82 \mathrm{a}$ & $25,58 \mathrm{a}$ & $8,58 \mathrm{ab}$ & $4,19 \mathrm{a}$ & $12,77 \mathrm{a}$ \\
\hline & & Média & $8,91 \mathrm{~A}$ & $25,03 \mathrm{~A}$ & $8,48 \mathrm{~A}$ & $4,42 \mathrm{~A}$ & $12,90 \mathrm{~A}$ \\
\hline Tradicional $^{3 /}$ & - & - & 7,54 & 22,91 & 7,19 & 4,34 & 11,52 \\
\hline
\end{tabular}

Em que: ${ }^{1 /}$ formulação $15-09-12$ (Mg: 1\%, S: 2,3\%, B: 0,02\%, Cu: 0,05\%, Fe: 1\%, Mn: 0,06\%, Mo: 0,02\%, Zn: 0,05\%), com liberação total dos nutrientes de aproximadamente $5-6$ meses; ${ }^{2}$ formulação $13-06-16(1,4 \% \mathrm{Mg}, 10 \% \mathrm{~S}, 0,02 \% \mathrm{~B}$, $0,05 \% \mathrm{Cu}, 0,26 \% \mathrm{Fe}, 0,06 \% \mathrm{Mn}, 0,02 \% \mathrm{Mo}$ ), com liberação total dos nutrientes de aproximadamente três meses);

${ }^{3 /}$ Mudas produzidas em sacos plásticos durante o período de pré-viveiro (método tradicional).

Ainda, dentre os ALL utilizados, o Basacote mini foi mais eficiente que o Osmocote ${ }^{\circledR}$ neste período de pré-viveiro, possivelmente por possuir o tempo de liberação total dos nutrientes de cerca de três meses enquanto o Osmocote ${ }^{\circledR}$ de 5-6 meses. Em média, o crescimento das plantas produzidas em tubetes utilizando 
ALL foi superior ao das produzidas em sacos plásticos (método tradicional), mostrando o excelente potencial para produção de mudas de dendezeiro em tubetes.

Para cada variável, médias seguidas de mesma letra minúscula na coluna não diferem entre si a $5 \%$ de probabilidade pelo teste Tukey e médias seguidas de mesma letra maiúscula, na coluna, também não diferem entre si.

TABELA 2: Resumo da análise de variância para as variáveis diâmetro do coleto, altura, matéria seca da parte aérea (MSPA), das raízes (MSR) e total (MST) de mudas de dendezeiro (Elaeis guineensis Jacq.) produzidas em tubetes plásticos de $120 \mathrm{~cm}^{3}$, aos três meses de pré-viveiro.

TABLE 2: Analysis of variance for diameter, height and aboveground, roots and total dry matter of oil palm seedlings (Elaeis guineensis Jacq.) produced in plastic containers, evaluated after three months of pre-nursery.

\begin{tabular}{|c|c|c|c|c|c|c|}
\hline Fonte de Variação & GL & Diâmetro & Altura & MSPA & MSR & MST \\
\hline Bloco & 3 & $2,59^{\text {ns }}$ & $0,20^{\text {ns }}$ & $2,98^{\mathrm{ns}}$ & $1,24^{\mathrm{ns}}$ & $1,56^{\text {ns }}$ \\
\hline Ocupação da bandeja (OB) & 3 & $2,08^{\text {ns }}$ & $1,72^{\mathrm{ns}}$ & $1,82^{\mathrm{ns}}$ & $3,99^{*}$ & $3,61^{*}$ \\
\hline Dose adubo (DA) & 1 & $107,22^{* *}$ & $89,66^{* *}$ & $249,82^{* *}$ & $22,96^{* *}$ & $261,39^{* *}$ \\
\hline Tipo de adubo (TA) & 1 & $6,11^{*}$ & $0,42^{\mathrm{ns}}$ & $3,77^{\mathrm{ns}}$ & $3,55^{\mathrm{ns}}$ & $8,91^{* *}$ \\
\hline $\mathrm{OB} \times \mathrm{DA}$ & 3 & $1,26^{\mathrm{ns}}$ & $5,13^{* *}$ & $4,36^{* *}$ & $2,33^{\mathrm{ns}}$ & $5,87^{* *}$ \\
\hline $\mathrm{OB} \times \mathrm{TA}$ & 3 & $2,19^{\mathrm{ns}}$ & $0,21^{\mathrm{ns}}$ & $2,59^{\mathrm{ns}}$ & $0,85^{\mathrm{ns}}$ & $1,05^{\text {ns }}$ \\
\hline DA $x$ TA & 1 & $6,11^{*}$ & $0,42^{\mathrm{ns}}$ & $3,77^{\mathrm{ns}}$ & $3,55^{\mathrm{ns}}$ & $8,91^{* *}$ \\
\hline OB $x$ DA $x$ TA & 3 & $2,19^{\text {ns }}$ & $0,21^{\mathrm{ns}}$ & $2,59^{\mathrm{ns}}$ & $0,85^{\mathrm{ns}}$ & $1,05^{\mathrm{ns}}$ \\
\hline Resíduo & 45 & & & & & \\
\hline CV (\%) & & 9,9 & 11,8 & 16,7 & 29 & 13,6 \\
\hline
\end{tabular}

Em que: $* *$ e $*=$ significativo a $1 \%$ e $5 \%$ de probabilidade, respectivamente; ns $=$ não significativo a $5 \%$ de probabilidade.

Observou-se que os teores de nutrientes das mudas produzidas em tubetes foram bastante afetados pela aplicação dos ALL, sobretudo para $\mathrm{N}$ e $\mathrm{K}$, na parte aérea, e para $\mathrm{K}$ nas raízes (Tabela 3). Apesar de possuir boa concentração destes nutrientes, a quantidade fornecida para as mudas pelo substrato foi muito pequena, em função de seu pequeno volume explorado $\left(120 \mathrm{~cm}^{3}\right)$, o que proporcionou pequena quantidade fornecida, insuficiente para melhor crescimento das mudas sem adubação. O potássio está envolvido na fotossíntese e, na sua carência, verifica-se redução na taxa fotossintética por unidade de área foliar e também maior taxa de respiração. A combinação desses fatores pode reduzir as reservas de carboidratos da planta (PRETTY, 1982; MARSCHNER, 1995). Um suprimento inadequado de potássio também faz com que os estômatos não se abram regularmente, podendo ocorrer menor assimilação de $\mathrm{CO}_{2}$ nos cloroplastos, diminuindo, consequentemente, a taxa fotossintética e o crescimento e produção de matéria seca das plantas. Esses resultados obtidos estão de acordo com Pacheco et al. (1987) e Botelho et al. (1998), que verificaram que N, $\mathrm{P}$ e $\mathrm{K}$ foram indispensáveis para maior crescimento de mudas de dendê e melhoria do estado nutricional.

Verificou-se que, assim como para o teor, o conteúdo de nutrientes foi superior em mudas produzidas em tubete com ALL comparado às plantas produzidas em tubetes sem adubos, durante o período de préviveiro (Tabela 4). Em média, o conteúdo total de $\mathrm{N}, \mathrm{P}, \mathrm{K}, \mathrm{Ca}$ e $\mathrm{Mg}$ foi superior com a utilização do Osmocote ${ }^{\circledR}$ e do Basacote mini em relação às plantas produzidas em tubetes sem adubos. Em relação à testemunha, o efeito mais marcante foi para o magnésio e potássio. Possivelmente, os maiores valores obtidos para a produção de matéria seca total, durante a fase de pré-viveiro, utilizando o Osmocote ${ }^{\circledR}$ (Tabela 1) possam ser devidos ao fornecimento mais rápido de nutrientes (até três meses), em especial de $\mathrm{N}$ e $\mathrm{K}$, em relação ao Basacote mini (Tabela 4). Yamanishi et al. (2004), estudando a utilização de ALL na formação de mudas de mamoeiro, verificaram que tratamentos constituídos de ALL proporcionaram bom desenvolvimento às mudas e foram superiores aos com formulado NPK (14-14-14) em relação à maioria das características analisadas. 
TABELA 3: Concentração de nutrientes na parte aérea e nas raízes de mudas de dendezeiro (Elaeis guineensis Jacq.), aos 3 meses de pré-viveiro, em função do tipo (TA) e quantidades (Dose) de adubo de liberação lenta e da percentagem de ocupação das bandejas pelos tubetes (OB).

TABLE 3: Concentration of nutrients in aboveground and in the roots of oil palm seedlings (Elaeis guineensis Jacq.), evaluated after three months of pre-nursery, in response to the type and dosages of slow release fertilizer and of the percentage of tray occupation by the containers.

\begin{tabular}{|c|c|c|c|c|c|c|c|c|c|c|c|c|}
\hline \multirow{3}{*}{ TA } & \multirow{3}{*}{$\begin{array}{c}\text { Dose } \\
\mathrm{kg} / \mathrm{m}^{3}\end{array}$} & \multirow{3}{*}{$\begin{array}{c}\text { OB } \\
\%\end{array}$} & \multicolumn{5}{|c|}{ Parte Aérea } & \multicolumn{5}{|c|}{ Raízes } \\
\hline & & & $\mathrm{N}$ & $\mathrm{P}$ & $\mathrm{K}$ & $\mathrm{Ca}$ & $\mathrm{Mg}$ & $\mathrm{N}$ & $\mathrm{P}$ & $\mathrm{K}$ & $\mathrm{Ca}$ & $\mathrm{Mg}$ \\
\hline & & & \multicolumn{10}{|c|}{$\mathrm{g} / \mathrm{kg}$} \\
\hline- & 0 & 100 & 9,90 & 1,82 & 11,95 & 6,38 & 5,78 & 6,03 & 2,00 & 8,81 & 2,24 & 2,58 \\
\hline - & 0 & 66 & 8,42 & 1,77 & 12,73 & 5,79 & 5,51 &, 60 & 2,33 & 8,63 & 2,28 & 2,35 \\
\hline- & 0 & 50 & 9,97 & 1,81 & 10,30 & 6,39 & 5,69 & 5,99 & 2,17 & 8,22 & 2,23 & 2,64 \\
\hline & 0 & 25 & 9,67 & 2,09 & 14,07 & 5,83 & 5,33 & 5,76 & 2,37 & 8,87 & 2,33 & 2,55 \\
\hline & & Média & 9,49 & 1,87 & 12,26 & 6,10 & 5,57 & 5,84 & 2,21 & 8,63 & 2,27 & 2,53 \\
\hline \multirow{5}{*}{ Osmocote $\mathbb{\circledR}^{1 /}$} & 3 & 100 & 17,37 & 1,75 & 15,60 & 7,15 & 5,32 & 8,63 & 1,38 & 15,42 & 2,60 & 2,45 \\
\hline & 3 & 66 & 16,56 & 1,59 & 14,53 & 6,87 & 5,14 & 8,09 & 1,23 & 14,75 & 2,58 & 2,49 \\
\hline & 3 & 50 & 16,17 & 1,71 & 15,03 & 7,08 & 5,32 & 7,93 & 1,31 & 15,03 & 2,38 & 2,32 \\
\hline & 3 & 25 & 16,11 & 1,69 & 14,14 & 6,90 & 5,37 & 7,96 & 1,34 & 13,78 & 2,45 & 2,47 \\
\hline & & Média & 16,55 & 1,68 & 14,82 & 7,00 & 5,29 & 8,15 & 1,31 & 14,74 & 2,50 & 2,43 \\
\hline \multirow{5}{*}{ Basacote mini ${ }^{2 /}$} & J & 100 & 13,33 & 2,09 & 18,08 & 7,07 & 4,59 & 6,86 & 2,08 & 17,65 & 2,00 & 1,86 \\
\hline & 3 & 66 & 13,37 & 1,91 & 17,34 & 7,78 & 4,70 & 6,07 & 1,77 & 15,85 & 1,88 & 1,82 \\
\hline & 3 & 50 & 11,70 & 1,97 & 18,85 & 6,97 & 4,61 & 6,25 & 1,59 & 16,56 & 1,87 & 1,78 \\
\hline & 3 & 25 & 11,96 & 2,00 & 18,34 & 7,03 & 4,60 & 6,45 & 1,94 & 15,08 & 1,97 & 1,81 \\
\hline & & Média & 12,59 & 1,99 & 18,15 & 7,21 & 4,62 & 6,41 & 1,84 & 16,28 & 1,93 & 1,82 \\
\hline Tradicional $^{3 /}$ & 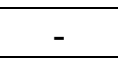 & - & 16,59 & 1,44 & 9,00 & 5,71 & 1,62 & 8,78 & 0,96 & 7,72 & 1,37 & 1,08 \\
\hline
\end{tabular}

Em que: ${ }^{1 /}$ formulação $15-09-12$ (Mg: 1\%, S: 2,3\%, B: 0,02\%, Cu: 0,05\%, Fe: 1\%, Mn: 0,06\%, Mo: 0,02\%, Zn: 0,05\%), com período de liberação total dos nutrientes de aproximadamente $5-6$ meses; ${ }^{2 /}$ formulação $13-06-16(1,4 \% \mathrm{Mg}, 10 \% \mathrm{~S}$, $0,02 \% \mathrm{~B}, 0,05 \% \mathrm{Cu}, 0,26 \% \mathrm{Fe}, 0,06 \% \mathrm{Mn}, 0,02 \% \mathrm{Mo}$ ), com período de liberação total dos nutrientes de aproximadamente 3 meses); ${ }^{3 /}$ Mudas produzidas em sacos plásticos durante o período de pré-viveiro (método tradicional).

Os valores obtidos para altura e diâmetro do coleto das plantas aos 10 meses de idade, sendo sete meses de viveiro e três de pré-viveiro, encontram-se na Tabela 5. Apesar de no período de pré-viveiro não terem sido verificadas diferenças estatisticamente significativas entre as alturas médias das plantas adubadas com ALL (Tabela 1), aos sete meses de viveiro, as plantas adubadas com o Osmocote ${ }^{\circledR}$ apresentaram maiores valores comparados aos demais tratamentos. Possivelmente, isso pode ser atribuído ao fato de o Osmocote ${ }^{\circledR}$ ter um tempo maior de liberação dos nutrientes (5-6 meses) comparativamente ao Basacote mini (três meses) propiciando fornecimento mais uniforme dos nutrientes ao longo do tempo. $\mathrm{O}$ fator percentagem de ocupação das bandejas pelos tubetes, durante os três meses de pré-viveiro, não influenciou o crescimento das plantas no viveiro aos 10 meses de idade (Tabela 5). As mudas produzidas em tubetes plásticos sem adubação apresentaram crescimento médio inferior às produzidas em tubetes com ALL e pelo método tradicional (Tabela 5). 
TABELA 4: Quantidade acumulada de nutrientes na parte aérea e total (parte aérea + raízes) de mudas de dendezeiro (Elaeis guineensis Jacq.), aos três meses de pré-viveiro, em função do tipo (TA) e doses do adubo de liberação lenta e da percentagem de ocupação das bandejas pelos tubetes (OB).

TABLE 4: Aboveground and total nutrient content of oil palm seedlings (Elaeis guineensis Jacq.), evaluated after three months of pre-nursery, in response to the type and dosages of the slow release fertilizer and of the percentage of tray occupation by the containers.

\begin{tabular}{|c|c|c|c|c|c|c|c|c|c|c|c|c|}
\hline \multirow{3}{*}{ TA } & \multirow{2}{*}{ Dose } & \multirow{2}{*}{ OB } & \multicolumn{5}{|c|}{ Parte Aérea } & \multicolumn{5}{|c|}{ Total } \\
\hline & & & $\mathrm{N}$ & $P$ & $\mathrm{~K}$ & $\mathrm{Ca}$ & $\mathrm{Mg}$ & $\mathrm{N}$ & $\mathrm{P}$ & K & $\mathrm{Ca}$ & $\mathrm{Mg}$ \\
\hline & $\mathrm{kg} / \mathrm{m}^{3}$ & $\%$ & \multicolumn{10}{|c|}{$\mathrm{mg} /$ planta } \\
\hline- & 0 & 100 & 42,03 & 7,71 & 49,13 & 27,41 & 24,55 & 61,25 & 14,07 & 76,77 & 34,54 & 32,77 \\
\hline - & 0 & 66 & 28,83 & 6,17 & 43,65 & 19,81 & 18,88 & 38,53 & 10,28 & 59,33 & 23,88 & 23,27 \\
\hline - & 0 & 50 & 49,28 & 8,78 & 47,40 & 32,03 & 28,01 & 69,95 & 16,23 & 75,65 & 39,73 & 37,11 \\
\hline- & 0 & 25 & 34,48 & 7,41 & 49,96 & 20,80 & 18,97 & 50,12 & 13,83 & 73,86 & 27,20 & 25,95 \\
\hline & \multicolumn{2}{|c|}{ Média } & 38,66 & 7,52 & 47,53 & 25,01 & 22,60 & 54,97 & 13,60 & 71,40 & 31,34 & 29,77 \\
\hline \multirow{6}{*}{ Osmocote $\mathbb{R}^{1 /}$} & 3 & 100 & 127,83 & 12,88 & 114,93 & 52,85 & 39,33 & 151,36 & 16,65 & 157,30 & 59,96 & 46,04 \\
\hline & 3 & 66 & 132,01 & 12,71 & 115,69 & 54,76 & 41,12 & 158,25 & 16,73 & 163,63 & 63,18 & 49,29 \\
\hline & 3 & 50 & 130,58 & 13,84 & 121,96 & 57,52 & 43,01 & 158,03 & 18,33 & 173,72 & 65,76 & 51,01 \\
\hline & 3 & 25 & 105,21 & 10,93 & 91,80 & 45,16 & 34,95 & 141,01 & 16,82 & 154,13 & 56,15 & 45,98 \\
\hline & \multicolumn{2}{|c|}{ Média } & 123,91 & 12,59 & 111,09 & 52,57 & 39,61 & 152,16 & 17,13 & 162,19 & 61,26 & 48,08 \\
\hline & 3 & 100 & 125,77 & 19,84 & 171,14 & 67,29 & 43,53 & 154,39 & 28,51 & 244,37 & 75,67 & 51,31 \\
\hline \multirow[t]{4}{*}{ Basacote mini ${ }^{2 /}$} & 3 & 66 & 121,67 & 17,29 & 157,12 & 70,63 & 42,59 & 146,74 & 24,61 & 222,26 & 78,35 & 50,07 \\
\hline & 3 & 50 & 79,21 & 13,27 & 127,30 & 46,80 & 3 & 111 & 21,64 & 216,09 & 56,60 & 40,48 \\
\hline & 3 & 25 & 102,46 & 17,13 & 156,57 & 60,17 & 39,29 & 129,50 & 25,28 & 219,17 & 68,46 & 46,85 \\
\hline & \multicolumn{2}{|c|}{ Média } & 107,28 & 16,88 & 153,03 & 61,22 & 39,14 & 135,60 & 25,01 & 225,47 & 69,77 & 47,18 \\
\hline radicional $^{3 /}$ & - & - & 118,99 & 10,44 & 64,17 & 41,13 & 11,62 & 157,02 & 14,58 & 97,36 & 47,02 & 16,29 \\
\hline
\end{tabular}

Em que: ${ }^{1 /}$ formulação 15-09-12 (Mg: 1\%, S: 2,3\%, B: 0,02\%, Cu: 0,05\%, Fe: 1\%, Mn: 0,06\%, Mo: 0,02\%, Zn: 0,05\%), com liberação dos nutrientes de aproximadamente $5-6$ meses; ${ }^{2 /}$ formulação $13-06-16(1,4 \% \mathrm{Mg}, 10 \% \mathrm{~S}, 0,02 \% \mathrm{~B}$, $0,05 \% \mathrm{Cu}, 0,26 \% \mathrm{Fe}, 0,06 \% \mathrm{Mn}, 0,02 \% \mathrm{Mo}$ ), com liberação dos nutrientes de aproximadamente três meses); ${ }^{3 /}$ Mudas produzidas em sacos plásticos durante o período de pré-viveiro. 
TABELA 5: Altura e diâmetro do coleto de mudas de dendezeiro (Elaeis guineensis Jacq.), aos 10 meses de idade (sete meses de viveiro), em função do tipo (TA) e doses de adubo de liberação lenta e da percentagem de ocupação das bandejas pelos tubetes (OB) durante o período de pré-viveiro.

TABLE 5: Height and diameter of oil palm seedlings (Elaeis guineensis Jacq.) evaluated after 10 months (seven months of nursery), in response to the type and dosages of slow release fertilizer and of the percentage of tray occupation by containers during pre-nursery.

\begin{tabular}{ccccc}
\hline TA & $\begin{array}{c}\text { Dose } \\
\mathrm{kg} / \mathrm{m}^{3}\end{array}$ & $\begin{array}{c}\text { OB } \\
\%\end{array}$ & $\begin{array}{c}\text { Altura } \\
\mathrm{cm}\end{array}$ & $\begin{array}{c}\text { Diâmetro } \\
\mathrm{mm}\end{array}$ \\
\hline- & 0 & 100 & $75,58 \mathrm{a}$ & $52,36 \mathrm{a}$ \\
- & 0 & 66 & $72,13 \mathrm{a}$ & $49,40 \mathrm{a}$ \\
- & 0 & 50 & $75,00 \mathrm{a}$ & $51,36 \mathrm{a}$ \\
- & 0 & 25 & $72,30 \mathrm{a}$ & $47,58 \mathrm{a}$ \\
\hline & & Média & $73,75 \mathrm{C}$ & $50,17 \mathrm{C}$ \\
\hline & 3 & 100 & $85,85 \mathrm{a}$ & $60,46 \mathrm{a}$ \\
Osmocote $^{1 /}{ }^{1 /}$ & 3 & 66 & $87,83 \mathrm{a}$ & $62,72 \mathrm{a}$ \\
& 3 & 50 & $86,40 \mathrm{a}$ & $60,20 \mathrm{a}$ \\
& 3 & 25 & $88,70 \mathrm{a}$ & $62,40 \mathrm{a}$ \\
\hline & 3 & 100 & $81,50 \mathrm{a}$ & $59,82 \mathrm{a}$ \\
\hline${\text { Basacote } \text { mini }^{2 /}}^{2 /}$ & 3 & 66 & $81,88 \mathrm{a}$ & $58,80 \mathrm{a}$ \\
& 3 & 50 & $80,43 \mathrm{a}$ & $56,89 \mathrm{a}$ \\
& 3 & 25 & $85,73 \mathrm{a}$ & $60,30 \mathrm{a}$ \\
\hline Tradicional $^{1 /}$ & 0 & - & 91,60 & 65,10
\end{tabular}

Em que: ${ }^{1 /}$ formulação 15-09-12 (Mg: 1\%, S: 2,3\%, B: 0,02\%, Cu: 0,05\%, Fe: 1\%, Mn: 0,06\%, Mo: 0,02\%, Zn: 0,05\%), com liberação total dos nutrientes de aproximadamente 5-6 meses; ${ }^{2}$ formulação $13-06-16(1,4 \% \mathrm{Mg}, 10 \% \mathrm{~S}, 0,02 \% \mathrm{~B}$, $0,05 \% \mathrm{Cu}, 0,26 \% \mathrm{Fe}, 0,06 \% \mathrm{Mn}, 0,02 \% \mathrm{Mo}$ ), com liberação total dos nutrientes de aproximadamente três meses);

${ }^{3 /}$ Mudas produzidas em sacos plásticos durante o período de pré-viveiro (método tradicional).

Verificou-se que aos 16 meses de idade (13 meses de viveiro), o crescimento em altura e diâmetro e a produção de matéria seca não foram influenciados pelo tipo de ALL utilizado durante a fase de pré-viveiro (Tabela 6). Praticamente não houve interação entre os fatores estudados para nenhuma das variáveis analisadas, com exceção da interação $\mathrm{OB}$ x DA para o crescimento em altura das mudas. $\mathrm{O}$ fator $\mathrm{OB}$ influenciou a matéria seca de folíolos, de folhas, da parte aérea e total e não influenciou o crescimento em altura e diâmetro. Resultados semelhantes foram obtidos por Teixeira et al. (2006), que não observaram diferença no crescimento em altura e diâmetro, aos 16 meses de idade, em mudas de dendezeiro produzidas sem adubação de base em diferentes densidades na bandeja durante a fase de pré-viveiro. De modo geral, a ocupação de $100 \%$ da bandeja pelos tubetes tendeu a apresentar os maiores valores médios para todas as variáveis analisadas (Tabela 7), logo o uso de $100 \%$ de ocupação durante os três meses de pré-viveiro assume grande importância, pois se pode otimizar a área ocupada, comparativamente aos demais tratamentos $(66,50$ e $25 \%)$, produzindo maior número de mudas por unidade de área de pré-viveiro. Entretanto, para períodos maiores que três meses de pré-viveiro, se for o caso, a melhor densidade de utilização das bandejas deve ser avaliada, pois o crescimento pode ser influenciado pela morfologia e velocidade de crescimento das mudas. 
TABELA 6: Resumo da análise de variância (Quadrado médio) para as variáveis altura, diâmetro do coleto, matéria seca de folíolos, de folhas, do caule, de raízes, da parte aérea, das raízes e total de mudas de dendezeiro (Elaeis guineensis Jacq.), aos 16 meses de idade, em função da ocupação da bandeja pelos tubetes (OB) e do tipo e dose de adubos de liberação lenta.

TABLE 6: Analysis of variance for height, diameter, and leaflets, leaves, stem, aboveground, roots and total dry matter of oil palm seedlings (Elaeis guineensis Jacq.), evaluated after 16 months, in response to the tray occupation by containers and to the type and dosages of slow release fertilizers.

\begin{tabular}{lcrrrrrrrrrr}
\hline Fontes de Variação & GL & Altura & Diâmetro & Folíolo & Folhas & Caule & Parte & Raízes & Total \\
\hline Bloco & 3 & $258,8^{* *}$ & $0,0247^{\text {ns }}$ & $17551^{* *}$ & $2316^{\text {ns }}$ & $7092^{\text {ns }}$ & $4558^{\text {ns }}$ & $1996^{\text {ns }}$ & $10565^{\text {ns }}$ \\
OB & 3 & $84,2^{\text {ns }}$ & $0,2629^{\text {ns }}$ & $11623^{* *}$ & $27580^{*}$ & $25056^{\text {ns }}$ & $98460^{*}$ & $848^{\text {ns }}$ & $103326^{*}$ \\
Dose adubo (DA) & 1 & $298,3^{* *}$ & $7,7820^{* *}$ & $36010^{* *}$ & $16451^{* *}$ & $27306^{* *}$ & $320140^{* *}$ & $2961^{\text {ns }}$ & $384682^{* *}$ \\
Tipo de adubo (TA) & 1 & $23,6^{\text {ns }}$ & $0,3964^{\text {ns }}$ & $522^{\text {ns }}$ & $16592^{\text {ns }}$ & $20151^{\text {ns }}$ & $73316^{\text {ns }}$ & $1040^{\text {ns }}$ & $91825^{\text {ns }}$ \\
OB x DA & 3 & $213,9^{* *}$ & $0,3021^{\text {ns }}$ & $353^{\text {ns }}$ & $10112^{\text {ns }}$ & $28506^{\text {ns }}$ & $45287^{\text {ns }}$ & $842^{\text {ns }}$ & $51202^{\text {ns }}$ \\
OB x TA & 3 & $40,5^{\text {ns }}$ & $0,0868^{\text {ns }}$ & $1693^{\text {ns }}$ & $4037^{\text {ns }}$ & $7584^{\text {ns }}$ & $8160^{\text {ns }}$ & $158^{\text {ns }}$ & $9019^{\text {ns }}$ \\
DA x TA & 1 & $23,6^{\text {ns }}$ & $0,3964^{\text {ns }}$ & $522^{\text {ns }}$ & $16593^{\text {ns }}$ & $20151^{\text {ns }}$ & $73316^{\text {ns }}$ & $1040^{\text {ns }}$ & $91025^{\text {ns }}$ \\
OB x DA x TA & 3 & $40,5^{\text {ns }}$ & $0,0868^{\text {ns }}$ & $1693^{\text {ns }}$ & $4037^{\text {ns }}$ & $7584^{\text {ns }}$ & $8160^{\text {ns }}$ & $158^{\text {ns }}$ & $9019^{\text {ns }}$ \\
Resíduo & 45 & 47,7 & 0,4031 & 2491 & 7282 & 14571 & 25441 & 838 & 27555 \\
CV $\%)$ & & 4,26 & 7,54 & 22,2 & 19,8 & 40,5 & 21,9 & 22,6 & 19,4 \\
\hline
\end{tabular}

Em que: $1 /$ Folhas $=$ matéria seca de folíolos + pecíolo + ráquis .

Em média, as plantas produzidas com a utilização dos ALL na dose de $3 \mathrm{~kg} / \mathrm{m}^{3}$ apresentaram crescimento e produção de matéria seca estatisticamente superiores que as produzidas sem adubação (Tabela 7). Assim, para o uso de tubetes na formação de mudas de dendezeiro, recomenda-se que seja feita a colocação de adubos no substrato durante a fase de pré-viveiro para que as mudas tenham bom padrão de crescimento. Nesse caso, fazem-se necessários novos estudos para se definir as melhores dosagens dos ALL para aumento da eficiência da adubação das mudas de dendezeiro e minimização dos custos.

TABELA 7: Altura, diâmetro do coleto e matéria seca de folíolos, de folhas, do caule, da parte aérea, das raízes e total de mudas de dendezeiro (Elaeis guineensis Jacq.), aos 16 meses de idade (13 meses de viveiro), produzidas em sacos plásticos (Tradicional) e em tubetes plásticos de 120 $\mathrm{cm}^{3}$ com diferentes percentagens de ocupação da bandeja (OB) e doses de adubos de liberação lenta durante os três meses de pré-viveiro.

TABLE 7: Height, diameter and leaflets, leaves, stem, aboveground, roots and total dry matter of oil palm seedlings (Elaeis guineensis Jacq.), evaluated after 16 months (13 months in nursery), produced in polybags (Traditional) and in plastic containers of $120 \mathrm{~cm}^{3}$ with different percentages of tray occupation (OB) and dosages of slow release fertilizers during the three months of pre-nursery.

\begin{tabular}{|c|c|c|c|c|c|c|c|c|}
\hline \multirow{2}{*}{ Tratamentos } & Altura & Diâmetro & Folíolos & Folhas $^{1 /}$ & Caule & Parte Aérea & Raízes & Total \\
\hline & \multicolumn{2}{|c|}{$\mathrm{cm}$} & \multicolumn{6}{|c|}{$\mathrm{g} /$ planta } \\
\hline Tradicional & 177,6 & 9,6 & 247,5 & 502,3 & 283,4 & 785,7 & 188,6 & 974,3 \\
\hline \multicolumn{9}{|l|}{ OB } \\
\hline $100 \%$ & $164,1 \mathrm{~A}$ & $8,6 \mathrm{~A}$ & $250,3 \mathrm{~A}$ & $478,9 \mathrm{~A}$ & $318,8 \mathrm{~A}$ & $797,7 \mathrm{~A}$ & $138,4 \mathrm{~A}$ & $936,1 \mathrm{~A}$ \\
\hline $66 \%$ & $163,0 \mathrm{~A}$ & $8,2 \mathrm{~A}$ & $240,9 \mathrm{AB}$ & $448,8 \mathrm{AB}$ & $339,2 \mathrm{~A}$ & $788,0 \mathrm{AB}$ & $120,9 \mathrm{~A}$ & $908,9 \mathrm{AB}$ \\
\hline $50 \%$ & 158,9 A & $8,4 \mathrm{~A}$ & $189,8 \quad$ B & $382,6 \quad$ B & $249,8 \mathrm{~A}$ & $632,4 \quad \mathrm{~B}$ & $127,5 \mathrm{~A}$ & 759,9 В \\
\hline $25 \%$ & $161,0 \mathrm{~A}$ & $8,4 \mathrm{~A}$ & $218,0 \mathrm{AB}$ & $416,2 \mathrm{AB}$ & $282,3 \mathrm{~A}$ & $698,5 \mathrm{AB}$ & $126,9 \mathrm{~A}$ & $825,4 \mathrm{AB}$ \\
\hline \multicolumn{9}{|l|}{ Doses } \\
\hline $3 \mathrm{~kg} / \mathrm{m}^{3}$ & $168,6 \mathrm{~A}$ & $8,8 \mathrm{~A}$ & $248,5 \mathrm{~A}$ & $481,7 \mathrm{~A}$ & $318,1 \mathrm{~A}$ & $799,8 \mathrm{~A}$ & $135,2 \mathrm{~A}$ & $935,0 \mathrm{~A}$ \\
\hline $0 \mathrm{~kg} / \mathrm{m}^{3}$ & 154,9 B & $8,0 \quad \mathrm{~B}$ & $201,0 \quad$ B & $381,5 \quad \mathrm{~B}$ & $276,8 \mathrm{~A}$ & $658,4 \quad \mathrm{~B}$ & $121,6 \mathrm{~A}$ & $780,0 \quad \mathrm{~B}$ \\
\hline
\end{tabular}

Em que: 1 Folhas $=$ matéria seca de folíolos + pecíolo + ráquis. Para OB e Doses, médias seguidas de mesma letra na coluna não diferem entre si a 5\% de probabilidade pelo teste de Tukey. 


\section{CONCLUSÕES}

A adição de fertilizantes ao substrato em tubetes plásticos durante a fase de pré-viveiro foi fundamental para a formação final das mudas de dendezeiro;

A ocupação diferenciada das bandejas pelos tubetes durante a fase de pré-viveiro não influenciou o crescimento em altura e diâmetro das mudas de dendezeiro aos 10 e 16 meses de idade;

A altura das plantas produzidas em tubetes foi superior, aos 10 meses de idade, quando se adicionou o Osmocote ${ }^{\circledR}$, com liberação dos nutrientes de 5-6 meses, em relação ao Basacote mini, com liberação dos nutrientes de três meses. Entretanto, o crescimento final das mudas, aos 16 meses de idade, não foi influenciado pelo tipo de ALL utilizado, mas pela dose utilizada.

\section{AGRADECIMENTOS}

Os autores agradecem ao CNPq (Conselho Nacional de Desenvolvimento Científico e Tecnológico) e à Embrapa Transferência de Tecnologia/Escritório de Negócios da Amazônia, pelo apoio financeiro. Agradecem também ao Magno Benarróz e João de Deus, e à Concita e Michele, pela valiosa ajuda durante a condução do experimento e a realização das análises laboratoriais, respectivamente.

\section{REFERÊNCIAS BIBLIOGRÁFICAS}

ANDRADE NETO, A.; MENDES, A. N. G.; GUIMARÃES, P. T. G. Avaliação de substratos alternativos e tipos de adubação para a produção de mudas de cafeeiro (Coffea arabica L.) em tubetes. Ciência e Agrotecnologia, Lavras, v. 23, n. 2, p. 270-280, abr. 1999.

BARCELOS, E. et al. Produção de mudas de dendezeiro na Amazônia. Manaus: Embrapa Amazônia Ocidental, 2001. 11 p. (Circular Técnica, 8)

BOTElHO, S. M.; VIÉGAS, I. J. M.; SHU, E. Y. Doses de N, P e K na nutrição e no crescimento de mudas de dendezeiro. Belém: Embrapa Amazônia Oriental, 1998. 15 p. (Boletim de Pesquisa, 193)

CHEE, K. H.; CHIU, S. B.; CHAN, S. M. Pre-nursery seedlings grown on pot trays. The Planter, Kuala Lumpur, v. 73, n. 855, p. 295-299, 1997.

CHINCHILLA, C. M.; UMAÑA, C. H.; RICHARDSON, D. L. Material de desarrollo avanzado en viveros de palma aceitera (Elaeis guineensis Jacq.). I. Espaciamiento y volumen de bolsa. Turrialba, San José, v. 40, n. 4, p. 428-439, oto./dic. 1990.

CORLEY, R. H. V.; TINKER, P. B. The oil palm. 4th ed. Oxford: Blackwell Science, 2003. 562 p.

DOMINGUEZ-LERENA, S. et al. Container characteristics influence Pinus pinea seedling development in the nursery and field. Forest Ecology and Management, Amsterdam, v. 221, n. 1-3, p. 63-71, 2006.

FREITAS, T. A. S. et al. Mudas de eucalipto produzidas a partir de miniestacas em diferentes recipientes e substratos. Revista Árvore, Viçosa, v. 30, n. 4, p.519-528, jul./ago. 2006.

GONÇALVES, J.L.M. et al. Produção de mudas de espécies nativas: substrato, nutrição, sombreamento e fertilização. In: GONÇALVES, J. L. M.; BENEDETTI, V. Nutrição e fertilização florestal. Piracicaba: IPEF. 2000. p.309-350.

JINKS, R.; MASON, B. Effects of seedling density on the growth of Corsican pine (Pinus nigra var. maritima Melv.), Scots pine (Pinus sylvestris L.) and Douglas-fir (Pseudotsuga menziesii Franco) in containers. Annals of Forest Science, Champenoux, v. 55, p. 407-423, 1998.

Malavolta, E.; VITTI, G. C.; OLIVEIRA, S. A. Avaliação do estado nutricional das plantas: princípios e aplicações. Piracicaba: Potafós, 1997. 304 p.

MARSCHNER, H. Mineral nutrition of higher plants. London: Academic Press, 1995. 889p.

NEVES, J. C. L.; GOMES, J. M.; NOVAIS, R. F. Fertilização mineral de mudas de eucalipto. In: BARROS, N. F.; NOVAIS, R.F. (Eds.) Relação solo-eucalipto. Viçosa, Folha de Viçosa, 1990. p.99-126.

NUNES, C. D.; LIMA, D.; CUNHA, R. N. V. Germinação de sementes de dendê (Elaeis guineensis Jacq.), utilizando o método de calor seco. Manaus: Embrapa Amazônia Ocidental, 1998. 3 p. (Instrução Técnica, 12)

OLIVEIRA, P. S. R.; GUALBERTO, R.; FAVORETO, A. J. Efeito do osmocote adicionado ao substrato plantmax na produção de mudas de café em tubetes. In: CONGRESSO BRASILEIRO DE PESQUISAS CAFEEIRAS, 21., 1995, Caxambu. Anais... Caxambu: PROCAFÉ/DENAC, 1995. p.70-72.

OTTMAN, M. J.; WELCH, L. F. Planting patterns and radiation interception, plant nutrient concentration, and yield in corn. Agronomy Journal, Madison, v. 81, n. 2, p. 167-174, Mar./Apr. 1989.

PACHECO, A. R.; TAILLIEZ, B. J. Formação de mudas de dendê. Manaus: EMBRAPA-CNPSD, 1985. 
PACHECO, A. R.; TAILliEZ, B. J.; VIÉGAS, I. J. M. Resposta de N-P-K-Ca e Mg no desenvolvimento de mudas de dendê na região de Manaus-AM. Belém: Embrapa-UEPAE de Belém, 1987.

PRETTY, K. M. O potássio na qualidade dos produtos agrícolas. In: YAMADA, T. et al. (Eds.) Potássio na Agricultura Brasileira. Piracicaba: Instituto da Potassa e Fosfato, 1982. p.177-194.

SCHWENGBER, J. E. et al. Utilização de diferentes recipientes na propagação da ameixeira através de estacas. Revista Brasileira de Fruticultura, Jaboticabal, v. 24, n. 1, p. 285-288, jan./abr. 2002.

SIDHU, M.; SINURAYA, Z. Comparative evaluation of new fertilizer types for use in oil palm nurseries and young field plantings. The Planter, Kuala Lumpur, v. 76, n. 894, p. 537-562, 2000.

SIMÕES, J. W. Problemática da produção de mudas em essências florestais. Série Técnica IPEF, Piracicaba, v. 4, n. 13, p. 1-29, 1987.

TEIXEIRA, P. C. et al. Crescimento de mudas de dendezeiro (Elaeis guineensis Jacq.) produzidas em tubetes e em sacolas plásticas durante a fase de pré-viveiro.In: FERTBIO, 2006, Bonito. Anais... Bonito: Embrapa Agropecuária Oeste, 2006. 1CD-ROM.

TIMMIS, R.; TANAKA, Y. Effects of container density and plant water stress on growth and cold hardiness of Douglas-fir seedlings, Forest Science, Bethesda, v. 22, n. 2, p. 167-172, 1976.

YAMANISHI, O. K. et al. Efeito de diferentes substratos e duas formas de adubação na produção de mudas de mamoeiro. Revista Brasileira de Fruticultura, Jaboticabal, v. 26, n. 2, p. 276-279, maio/ago. 2004. 